\title{
Perirhinal and Postrhinal Contributions to Remote Memory for Context
}

\author{
Rebecca D. Burwell, David J. Bucci, Matthew R. Sanborn, and Michael J. Jutras \\ Departments of Psychology and Neuroscience, Brown University, Providence, Rhode Island 02912
}

The perirhinal (PER) and postrhinal (POR) cortices, two components of the medial temporal lobe memory system, are reciprocally connected with the hippocampus both directly and via the entorhinal cortex. Damage to PER or POR before or shortly after training on a contextual fear conditioning task causes deficits in the subsequent expression of contextual fear, implicating these regions in the acquisition or expression of contextual memory. Here, we examined the contribution of PER and POR to the processing of remotely learned contextual information. Male Long-Evans rats were trained in an unsignaled contextual fear conditioning paradigm. After training, rats received bilateral neurotoxic lesions to PER or POR or sham control surgeries at three different training-to-lesion intervals: 1, 28, or 100 d after training. Two weeks after surgery, lesioned and control rats were returned to the training context to assess contextual fear as measured by freezing. Rats with PER or POR damage froze significantly less in the training context than control rats but were not different from each other. The severity of the deficit did not differ across training-to-lesion intervals for any group. This pattern of deficits differs from that of posttraining hippocampal lesions, for which longer training-to-lesion intervals produce significantly more fear-conditioned contextual freezing than shorter training-to-lesion intervals. In the absence of such a retrograde gradient in the present study, our interpretation is that PER and POR have an ongoing role in the storage or retrieval of representations for context. Alternatively, these regions may be involved in a more extended consolidation process that becomes apparent beyond $100 \mathrm{~d}$ after learning.

Key words: fear conditioning; hippocampus; classical conditioning; memory; consolidation; parahippocampal

\section{Introduction}

Pavlovian fear conditioning has been widely used to examine hippocampal involvement in contextual learning and memory (for review, see Nadel and Willner, 1980; Maren et al., 1998; Holland and Bouton, 1999). Growing evidence suggests that the hippocampus is not the site of permanent memory storage for at least some types of memory (Winocur et al., 1987; Kim and Fanselow, 1992; Maren et al., 1997). Instead, investigators have proposed that long-term memories are stabilized and stored in the neocortex (McClelland et al., 1995; Eichenbaum, 1997; Anagnostaras et al., 2001; Nadel and Bohbot, 2001). Indeed, hippocampal damage at 1 or $14 \mathrm{~d}$ impairs preoperatively acquired contextual fear conditioning, but damage at $28 \mathrm{~d}$ (Kim and Fanselow, 1992), 50 d (Anagnostaras et al., 1999), or $100 \mathrm{~d}$ (Maren et al., 1997) results in significantly greater fearconditioned freezing to context compared with earlier time points. Thus, the hippocampus appears to have a time-limited

\footnotetext{
Received Sept. 12, 2004; revised 0ct. 19, 2004; accepted 0ct. 25, 2004.

This work was supported by a National Science Foundation Career Award (IBN 9875792) to R.D.B., Brown University Undergraduate Training and Research Assistantships to M.R.S. and M.J.J., and a National Institute of Mental Health Postdoctoral Fellowship (F32-MH12426) to D.J.B.

Correspondence should be addressed to Dr. Rebecca D. Burwell, Department of Psychology, Brown University, 89 Waterman Street, Providence, RI 02912. E-mail: Rebecca Burwell@Brown.edu.

D. J. Bucci's present address: Department of Psychological and Brain Sciences, Dartmouth College, Hanover, NH 03755.

M. R. Sanborn's present address: Georgetown University School of Medicine, Washington, DC 20057.

M. J. Jutras's present address: Emory University Program in Neuroscience, Atlanta, GA 30322.

DOI:10.1523/JNEUROSCI.3781-04.2004

Copyright $\odot 2004$ Society for Neuroscience $\quad$ 0270-6474/04/2411023-06\$15.00/0
}

role in the consolidation of contextual representations that are likely stored outside the hippocampus in cortical regions.

Two cortical regions of particular interest in this regard are the perirhinal (PER) and postrhinal (POR) cortices. The PER and POR are higher-order cortical association areas, each receiving substantial unimodal and polymodal association input (Burwell and Amaral, 1998b). Each area is also reciprocally connected with the hippocampus, both directly and via the entorhinal cortex (Kosel et al., 1983; Naber et al., 1997; Shi and Cassell, 1997; Burwell and Amaral, 1998a). Studies conducted in our laboratory and by others suggest that PER and POR are involved in the encoding or processing of contextual information (Corodimas and LeDoux, 1995; Bucci et al., 2000, 2002; Burwell et al., 2004). Lesions of either region made before or shortly after training resulted in deficits in contextual fear, but the role of PER and POR in contextual memory at longer time points after training has not been described thoroughly. The close reciprocal connectivity with the hippocampal formation suggests that the PER and POR may contribute directly to the hippocampal-dependent consolidation process. If that is the case, posttraining lesions should reveal a graded memory impairment similar to what has been described previously for the hippocampus. Alternatively, the PER and POR may have an ongoing role in processing contextual information. In that event, a graded memory impairment would not be evident.

We tested these predictions by examining the effects of PER or POR damage produced 1,28 , or $100 \mathrm{~d}$ after training on the retention of contextual fear. Similar levels of impairment at all 
training-to-surgery intervals for lesioned rats would suggest PER and POR have a prolonged role in the processing or retrieval of contextual information, possibly providing the locus of storage for contextual representations.

\section{Materials and Methods}

Experimental design. Sham surgeries and PER or POR lesions were conducted 1,28 , or $100 \mathrm{~d}$ after training. These intervals were chosen because they span the intervals for which hippocampal damage shows a retrograde memory gradient.

Subjects. Subjects were 88 male Long-Evans rats obtained from Charles River Laboratories (Wilmington, MA). All subjects weighed $300-350 \mathrm{gm}$ at the time of surgery. The rats were housed individually in wire mesh cages and maintained on a $12 \mathrm{hr}$ light/dark cycle with ad libitum access to food and water. Each rat was handled by the experimenter for 1-2 min each day for 1 week before the start of the experiment. Before training, all rats were habituated to a plastic transporter used for carrying rats from the colony room to the behavioral chambers. All methods for handling research animals were according to approved Institutional Animal Care and Use Committee and Association for Assessment and Accreditation of Laboratory Animal Care guidelines.

Surgery. Subjects were brought to and maintained at a surgical level of anesthesia with halothane gas delivered through a nose cone (David Kopf Instruments, Tujunga, CA). The head was secured in a Kopf stereotaxic apparatus with the head level between bregma and lambda. Under aseptic conditions, an incision was made to reveal the skull, and the skin was retracted to the side. A series of holes was then drilled through the skull above the intended lesion site. Bilateral neurotoxic lesions of the PER or POR were made using ibotenic acid ( $10 \mu \mathrm{g} / \mu \mathrm{l}$; Sigma, St. Louis, MO) dissolved in $0.1 \mathrm{M}$ sodium phosphate buffer (PB). Ibotenic acid was pressure injected into the brain through a glass pipette (50 $\mu \mathrm{m}$ tip). For PER lesions, injections of $0.025-0.05 \mu \mathrm{l}$ were made at: $2.3,3.3,4.3,5.2,6.4$, and $7.1 \mathrm{~mm}$ posterior to bregma; $6.3,6.4,6.5,6.6,6.7$, and $6.1 \mathrm{~mm}$ lateral from the midline; and 8.0, 8.0, 8.0, 7.0, 6.1, and $5.2 \mathrm{~mm}$ below the skull surface, respectively. For POR lesions, the pipette was angled at $22^{\circ}$ from vertical with the tip oriented rostrally and lowered through the skull at $2.0 \mathrm{~mm}$ posterior to lambda. An injection of $0.05 \mu \mathrm{l}$ was made at each of two sites: at $0.3 \mathrm{~mm}$ lateral from the lateral ridge at $5.92 \mathrm{~mm}$ below the skull surface and at $0.2 \mathrm{~mm}$ medial to the ridge at $4.85 \mathrm{~mm}$ below the skull surface. All injections were made at a rate of $0.033 \mu \mathrm{l} / \mathrm{min}$, and the pipette was left in place for $30 \mathrm{sec}$ before and $2 \mathrm{~min}$ after each injection. Rats were allowed to recover for 2 weeks before resuming behavioral training. For sham-lesioned control animals, rats were anesthetized, and holes were drilled as above, but no injections were made.

Apparatus. Behavioral training was conducted in an operant testing environment interfaced with a personal computer and controlled by the MED-PC version 2.1 software package (MED Associates, St. Albans, VT). Custom software written in the Pascal-based MED-PC notation controlled the behavioral tasks and recorded task events and responses. Experiments were conducted in four $24 \times 30.5 \times 29 \mathrm{~cm}$ operant test chambers with modular component aluminum panels in the front and back, Plexiglas side panels and top, and a floor constructed of $0.48 \mathrm{~cm}$ rods placed $1.6 \mathrm{~cm}$ apart. Scrambled alternating current was delivered through the grid floor by a constant-current shock source. A speaker connected to a programmable audio input generator was located at the top right corner of the front panel. A partially shaded house light $(28 \mathrm{~V}$; $100 \mathrm{~mA}$ ) mounted centrally at the top of the front wall illuminated the chamber during habituation, training, and testing. Each testing chamber was enclosed in a $62 \times 56 \times 56 \mathrm{~cm}$ sound-attenuating chamber fitted with an exhaust fan that provided air flow to the test chamber and background white noise. A video camera was mounted above and behind each chamber on the back wall of the sound-attenuating chamber, and a video cassette recorder was used to record behavior in all four chambers simultaneously.

Behavioral procedures. One day before behavioral training began, rats were habituated to a plastic transporter used for carrying rats from the colony room to the behavioral chambers. Rats were then trained in cohorts of two to four rats in an unsignaled Pavlovian contextual fear conditioning paradigm, as described previously (Bucci et al., 2000). Briefly, rats were placed in individual chambers on the training day and after 3 min received three constant-current shocks (1.0 mA; $1.0 \mathrm{sec} ; 64$ $\mathrm{sec}$ intertrial interval) delivered through the floor grid. Rats were removed from the chambers and returned to the colony room $64 \mathrm{sec}$ after the final shock was delivered and were randomly assigned to a lesion or control group. Surgery was conducted either $1 \mathrm{~d}$ ( $24 \mathrm{hr}), 28$, or $100 \mathrm{~d}$ after training. Two weeks after surgery, rats were returned to the conditioning chambers for an $8 \mathrm{~min}, 32 \mathrm{sec}$ extinction test, during which no shocks were delivered. Behavioral responses during the training and testing days were recorded on videotape for subsequent analysis.

Behavioral observation. Freezing served as the index of conditioned fear (Fanselow, 1980). On the training day, the incidence of freezing behavior was recorded every $8 \mathrm{sec}$ during the $64 \mathrm{sec}$ period before delivery of the first shock and during the $64 \mathrm{sec}$ periods after each trial (immediate postshock freezing). During a $64 \mathrm{sec}$ observation period, each animal was observed every $8 \mathrm{sec}$ (yielding a total of eight observations per period) and was recorded as either freezing or not freezing. On the test day, the 8 min, $32 \mathrm{sec}$ observation period was divided into $64 \mathrm{sec}$ blocks, and freezing behavior was again recorded every $8 \mathrm{sec}$. The frequency of freezing behavior was converted to a percentage of total observations. The observer was unaware of lesion-interval condition when scoring behavior.

Histology. At the end of behavioral training, subjects were deeply anesthetized with sodium pentobarbital (Nembutal; 100 mg/kg; Abbott Laboratories, Chicago, IL) and transcardially perfused with normal saline and $4 \%$ paraformaldehyde in $0.1 \mathrm{M} \mathrm{PB}$ at a rate of $35-40 \mathrm{ml} / \mathrm{min}$. After fixation, each brain was removed from the skull and postfixed for $6 \mathrm{hr}$ at $4^{\circ} \mathrm{C}$ in the same buffered paraformaldehyde solution. Brains were cryoprotected for $24-48 \mathrm{hr}$ at $4^{\circ} \mathrm{C}$ in a solution of $20 \%$ glycerol in $0.1 \mathrm{M} \mathrm{PB}$. Coronal brain sections were cut at $40 \mu \mathrm{m}$ on a freezing microtome. Sections were collected in four series for PER-lesioned brains and two series for POR-lesioned brains for subsequent processing and storage. One series was collected in a $10 \%$ formalin solution in preparation for cell staining. That series was subsequently mounted and stained for Nissl with thionin. The remaining series were stored in cryoprotectant tissuecollecting solution ( $30 \%$ ethylene glycol and $20 \%$ glycerol in $0.1 \mathrm{M} \mathrm{PB}$ ) at $-20^{\circ} \mathrm{C}$.

For histological analysis, camera lucida techniques were used to draw section contours, add regional borders, and circumscribe the location of tissue damage. Tissue damage was identified by marked thinning of the cortex, necrosis, or in some instances, missing tissue. Coronal sections were examined at $480 \mu \mathrm{m}$ intervals for PER lesions and at $240 \mu \mathrm{m}$ intervals for POR lesions. For each coronal section, areal measurements included the total area of the target region and the area of the target region that was damaged. According to previous studies, the extent of the lesion along the rostrocaudal axis is a good predictor of efficacy of the lesion (Bucci et al., 2000; Bucci and Burwell, 2004), which is consistent with the organization of cortical afferent and intrinsic connectivity of the PER and POR (Burwell and Amaral, 1998b). Thus, the proportion of sections in the rostrocaudal plane that exhibited damage was also quantified. A subject was retained in the study if a lesion involved distributed bilateral damage to the target region and did not include substantial bilateral damage to any region outside the target region.

Data analysis. Analysis of freezing behavior was conducted using repeated-measures ANOVA (rANOVA). Training data and extinction testing data were analyzed separately. For training, lesion condition and training-to-surgery interval were the between-subject variables and block was the within-subject variable. One time point before the first shock and three time points after each trial comprised four blocks during which freezing was assessed. No lesion or interval effects were expected, because training was conducted before surgery. For extinction testing, we analyzed percentage of freezing across eight 64 sec blocks. Lesion condition and interval were the between-subject variables and extinction block was the within-subject variable. Significant main effects and interactions were followed by appropriate comparisons. Correlation analyses were conducted on the two measures of lesion efficacy (i.e., proportion of area damaged and proportion of sections damaged). All analyses were conducted using SAS version 8.0 (SAS Institute, Cary, NC). An $\alpha$ level of 0.05 was adopted for all analyses. 


\begin{tabular}{|c|c|c|c|c|c|c|}
\hline \multirow[b]{2}{*}{ Surgery interval (days) } & \multicolumn{3}{|c|}{ Perirhinal lesions } & \multicolumn{3}{|c|}{ Postrhinal lesions } \\
\hline & $\begin{array}{l}\text { Percentage } \\
\text { of area }\end{array}$ & $\begin{array}{l}\text { Percentage } \\
\text { of sections }\end{array}$ & $n$ & $\begin{array}{l}\text { Percentage } \\
\text { of area }\end{array}$ & $\begin{array}{l}\text { Percentage } \\
\text { of sections }\end{array}$ & $n$ \\
\hline 1 & $21.2 \pm 4.8$ & $54.8 \pm 10.5$ & 8 & $27.1 \pm 7.8$ & $70.4 \pm 8.5$ & 7 \\
\hline 28 & $17.9 \pm 4.5$ & $46.8 \pm 7.7$ & 8 & $25.9 \pm 4.1$ & $74.5 \pm 5.6$ & 8 \\
\hline 100 & $16.2 \pm 4.1$ & $82.33 \pm 8.4$ & 6 & $38.0 \pm 6.4$ & $85.4 \pm 12.1$ & 9 \\
\hline Average & $18.9 \pm 2.7$ & $57.1 \pm 6.0$ & 22 & $30.1 \pm 3.6$ & $76.6 \pm 5.0$ & 24 \\
\hline
\end{tabular}
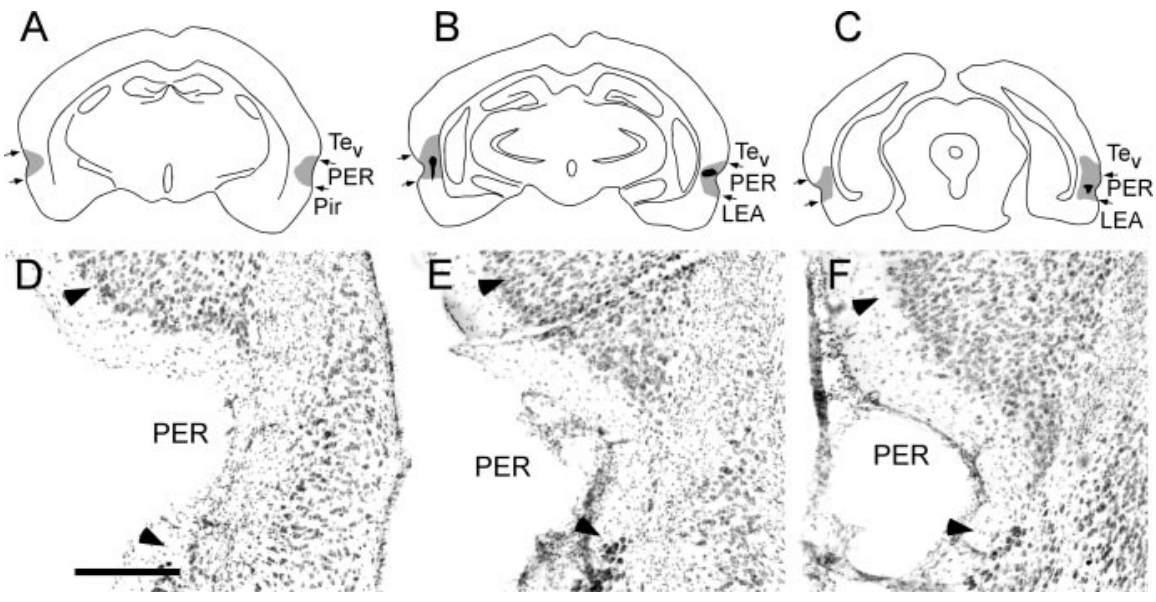

Figure 1. Perirhinal lesions. $A-C$, Schematic of the placement of the largest (gray) and smallest (black) PER neurotoxic lesion at three rostrocaudal levels, $-2.4,-3.8$, and $-6.7 \mathrm{~mm}$, according to bregma (Paxinos and Watson, 1998). Although only three levels are shown, it should be noted that the smaller lesion included damage observed in 5 of the 11 sections analyzed and was distributed bilaterally along the rostrocaudal axis. $D-F$, Photomicrograph of a representative PER neurotoxic lesion at three rostrocaudal levels. Arrowheads indicate cytoarchitectonic boundaries of PER. LEA, Lateral entorhinal cortex; Pir, piriform cortex; $\mathrm{Te}_{\mathrm{v}}$, temporal cortex. Scale bar, $300 \mu \mathrm{m}$.

\section{Results}

\section{Histology}

A total of 88 subjects were originally included in the experiment. One PER rat was eliminated as a result of little or no damage to the target or any other cortical region. One PER and five POR rats were eliminated because damage was primarily unilateral. One PER and one POR rat were eliminated because of substantial bilateral damage outside of the target region. Two PER rats died before extinction testing. For two PER rats and one POR rat, sections were missing from the case, precluding complete quantitative analysis; however, evaluation of the available sections indicated that lesions were comparable with those for other subjects in the present study (i.e., that there was extended bilateral damage to the target regions and little damage outside of the target areas). In the remaining lesioned subjects, damage produced by infusions of ibotenic acid into PER or POR was comparable with that observed in several previous studies (Bucci et al., 2000, 2002). Rats with acceptable POR lesions exhibited substantial bilateral damage to the target region. In most subjects, there was slight damage to the adjacent entorhinal or temporal association cortex. The hippocampus was spared in all lesioned subjects.

Our intention was to make small lesions that were well distributed across the rostrocaudal extent of the target regions to effectively compromise function of the region without damaging the hippocampus. Table 1 shows the mean percentage of areal damage and the mean percentage of total sections exhibiting damage for each lesion-interval group. The latter measure permits assessment of the distribution of damage. The percentage of area and the percentage of sections measures were highly intercorrelated $(r=0.78 ; p<0.0001)$. Both measures were negatively correlated, although not significantly, with freezing in the first four blocks of extinction testing for both lesion conditions (range, $r=$ -0.18 to $-0.31 ; p>0.14)$. The largest and smallest PER lesions are depicted in schematic form in Figure $1 A-C$. Figure $1 D-F$ shows a representative PER lesion in which marked thinning of cortex and necrosis was distributed through the rostrocaudal extent of the region, although damage to surface area was relatively small. The largest and smallest POR lesions are depicted in schematic form in Figure 2A-C. Figure $2 D-F$ shows a representative POR lesion.

\section{Behavior}

On the presurgical training day, rANOVA indicated that the level of postshock freezing was comparable for PER- and PORlesioned groups across all training-tolesion intervals (Fig. 3A,C,E). There was no main effect of lesion $(p>0.74)$, no main effect of interval $(p>0.12)$, and no lesion by interval interaction $(p>0.90)$. As would be expected, immediate postshock freezing increased with each successive conditioning trial $\left(F_{(3,198)}=233.53 ; p<0.0001\right)$.

During extinction testing 2 weeks after surgery, control animals froze more than the lesioned subjects overall (Fig. 3B,D,F). This was confirmed by a significant main effect of lesion $\left(F_{(2,68)}=\right.$ 8.77; $p<0.0003)$. There was no main effect of training-tosurgery interval $(p>0.51)$ and no lesion by interval interaction $(p>0.83)$. All groups exhibited a normal extinction curve. This was confirmed by within-subject analyses yielding a main effect of extinction block $\left(F_{(7,476)}=13.45 ; p<0.0001\right)$. There was no interaction of block with lesion $(p>0.62)$ or interval $(p>0.70)$ and no three-way interaction $(p>0.67)$.

Post hoc contrasts indicated that the sham control subjects froze significantly more than the PER subjects $(p<0.0002)$ and the POR subjects $(p<0.002)$. The PER- and POR-lesioned groups were not, however, significantly different from each other $(p>0.50)$.

Although there was no main effect of interval on extinction testing, it was of interest whether any group showed evidence of a retrograde gradient. Accordingly, we conducted additional post hoc analyses of each lesion condition, which confirmed that there was not a significant change across training-to-lesion interval. These analyses indicated that there was no main effect of interval or interval by extinction block interaction for the sham surgery group ( $p>0.77$ and 0.31 , respectively), the PER group ( $p>0.57$ and 0.92 ), or the POR group ( $p>0.65$ and 0.50 ). Thus, there was no evidence for either a retrograde gradient or forgetting for any of the three lesion conditions. 


\section{Discussion}

One view of episodic memory proposes that all structures within the medial temporal lobe cooperate in the temporary binding together of individual components of a memory trace distributed in neocortex. After a period of time, the memory becomes independent of medial temporal lobe structures in a process called consolidation (Squire and ZolaMorgan, 1991). Recently emerging viewpoints, however, propose that the medial temporal lobe comprises different memory subsystems that can be functionally dissociated (Eichenbaum et al., 1994; Aggleton and Brown, 1999; Maguire et al., 2000) and that the role of these structures in remote memory should be reconsidered (Murray and Bussey, 2001; Nadel and Moscovitch, 2001). Here, we examined the temporal role in contextual memory of two structures within the medial temporal area, PER and POR, using a classically conditioned fear paradigm combined with posttraining lesion methods. We showed that the PER and POR are involved in the storage, maintenance, or retrieval of the memory for contextual fear for at least $100 \mathrm{~d}$ after learning. There was no evidence for a temporally graded retrograde amnesia. These findings provide the first evidence of a prolonged role for POR in mnemonic processing and extend the results of previous studies of the temporal characteristics of PER memory function. This profile of deficits contrasts with that of the hippocampus, for which several studies have reported a consolidation gradient for contextually conditioned fear (Kim and Fanselow, 1992; Maren et al., 1997; Anagnostaras et al., 1999). Together, these findings support the notion that individual structures within the medial temporal lobe make different contributions to memory processes.

These effects of PER and POR damage cannot be explained by performance deficits, because rats with pretraining PER or POR damage are still able to exhibit freezing under other circumstances. Bucci et al. (2000) showed that pretraining electrolytic lesions spared freezing to a discrete conditioned stimulus (CS), a tone that signaled presentation of the shock, but disrupted freezing to the training context. In a second study using a contextual discrimination paradigm, rats with pretraining neurotoxic lesions of PER or POR were unable to discriminate between shock and no-shock contexts as reflected by substantial levels of freezing in both contexts (Bucci et al., 2002). The evidence suggests that rats with PER or POR damage are still able to encode the shock unconditioned stimulus (US) and to make associations between the US and discrete CSs. Thus, one interpretation of the present findings is that rats with PER or POR lesions have a selective deficit in the representation of context.

The present findings differ from some studies using inactivation methods. Infusions of tetrodotoxin (TTX) into rostral PER before (Sacchetti et al., 2002) and at time points up to $8 \mathrm{~d}$ after training, but not later (Sacchetti et al., 1999), impaired fearconditioned freezing to both context and an auditory CS. In those studies, TTX was infused to a single site, affecting only the most rostral part of the PER and probably including insular cortex. Notably, the location of this cortex is adjacent to the amygdala, which may have been affected by the inactivation. Indeed, in work by Sacchetti et al. (1999), posttraining inactivation of the basolateral amygdala up to $2 \mathrm{~d}$ after training also impaired subsequent expression of fear to context and to an auditory CS. Because impairment occurred in freezing to both the training context and the CS, it is reasonable to suggest that the representation of the shock US was disrupted in the studies of Sacchetti et al. $(1999,2002)$ either by disruption of insular cortex or by unintended inactivation of the amygdala. The location of inactivation may also explain the results of another study in which infusions of the $\mathrm{GABA}_{\mathrm{A}}$ agonist muscimol into rostral PER adjacent to the amygdala blocked the acquisition and expression of fearpotentiated startle (Schulz et al., 2004). Anterior perirhinal lesions also impaired the fear-potentiated startle to a visual CS (Rosen et al., 1992) and fear conditioning to olfactory CSs while sparing freezing to context (Herzog and Otto, 1997). Thus, aside from the possibility of unintended amygdala damage, it may be that the critical region for disruption of freezing to a discrete CS is the region surrounding the rhinal sulcus that is rostral to -2.3 $\mathrm{mm}$ relative to bregma. One exception is that conditioning to complex ultrasonic rat vocalizations used as the CS in a signaled fear conditioning paradigm was impaired by damage to the full rostrocaudal extent of PER (Lindquist et al., 2004). In that study, fear conditioning signaled by simple 4 and $22 \mathrm{kHz}$ tones was spared.

The absence of effects of PER damage on contextual fear in some studies may be attributable to the rostral location of the damage and sparing of caudal PER. We find that contextual fear conditioning or discrimination is spared unless the entire rostrocaudal extent of PER sustains damage. We use six small injections ranging from -2.3 to $-7.1 \mathrm{~mm}$ relative to bregma in each hemisphere (Bucci et al., 2000, 2002). Lesions are intentionally small to minimize the possibility of damage to the hippocampus or the amygdala while ensuring that normal function is disrupted in the entire rostrocaudal extent of the region. Thus, one interpretation that explains most of the available evidence is that posterior in- 


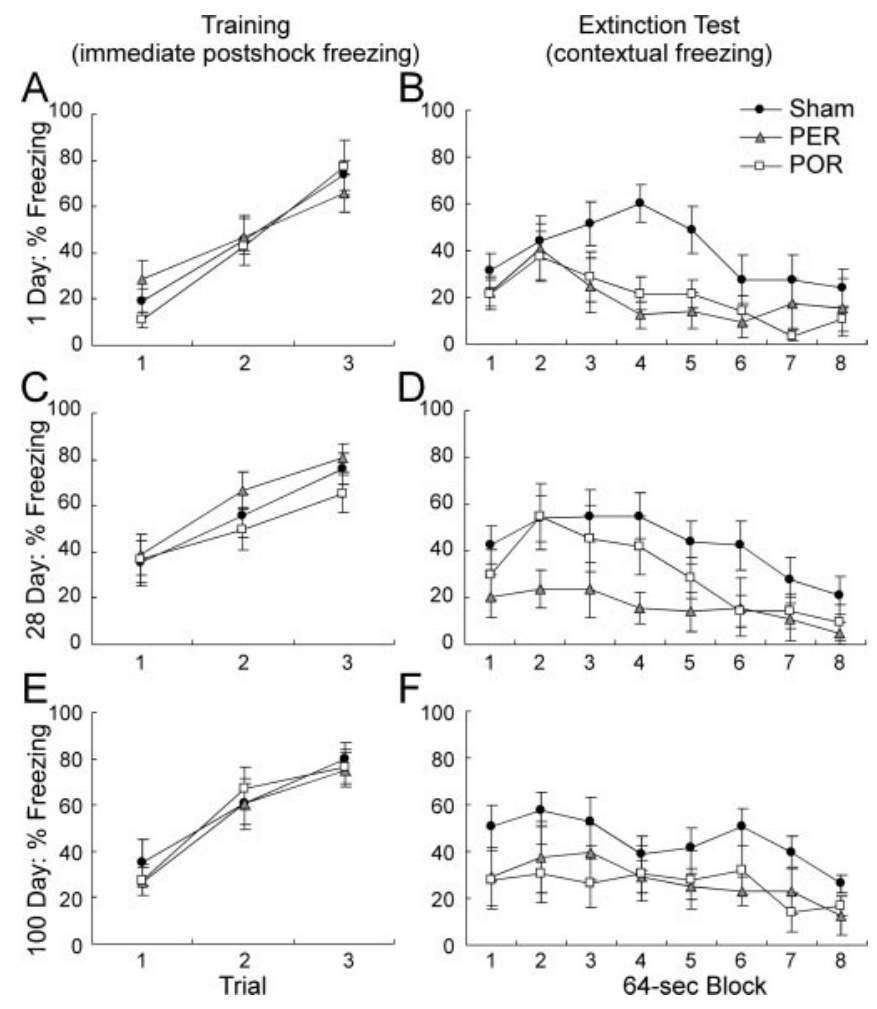

Figure 3. Percentage of freezing after training and during extinction tests. Training data are shown in the left panels, and extinction data are shown in the right panels for control subjects and rats receiving PER or POR lesions. Lesions were produced $1 \mathrm{~d}(A, B), 28 \mathrm{~d}(C, D)$, or $100 \mathrm{~d}(E$, $F$ ) after surgery. The training data are the percentage of immediate postshock freezing in the 64 sec after each trial. Extinction data are the percentage of freezing collapsed over the eight $64 \mathrm{sec}$ blocks of the extinction test. Data are mean \pm SEM.

sular cortex is involved in the representation of the US and that the PER and POR are involved in the representation of context.

What remains to be determined is the precise nature of the impairment to the representation of context resulting from extensive PER or POR damage. Are the observed deficits in the storage, retrieval, or consolidation of the representations for context? One possibility is that the complex, polymodal nature of context representations demands that they must necessarily be stored in a higher-order associational region such as the PER or POR. Another possibility is that context representations are stored in other neocortical regions, but that the PER and POR, situated between the hippocampal formation and neocortical sensory regions, are necessary for their retrieval. A third possibility is that these regions are still involved somehow in consolidation of representations of context. The lack of a retrograde gradient in the lesion conditions indicates that neither the PER nor the POR contribution diminishes within the first $100 \mathrm{~d}$ after learning. This leaves open the possibility that PER and POR are involved in a more extended consolidation process.

Previous studies suggest that the hippocampus is involved in the consolidation of information about context. Studies using electrolytic lesion methods have shown that damage to the hippocampus can result in temporally graded retrograde amnesia observable at training-to-lesion intervals of 28 and $50 \mathrm{~d}$ (Kim and Fanselow, 1992; Anagnostaras et al., 1999). Neurotoxic lesions of dorsal hippocampus showed severe retrograde memory deficits with posttraining lesions at 1 and $28 \mathrm{~d}$ but not at $100 \mathrm{~d}$ (Maren et al., 1997). Contextual freezing at testing when surgery followed training by $100 \mathrm{~d}$ was significantly greater than when surgery followed training by 1 or $28 \mathrm{~d}$, although a mild impairment was observed. This pattern of results differs from that of the present study using the same lesion methods and training-to-lesion intervals in which rats with PER or POR lesions exhibited similar levels of freezing to context at all intervals. Although it could be argued that the deficits in contextual fear conditioning reported here are the result of hippocampal disconnection from the PER and POR, if this were the case, damage to the PER or POR would show the same temporal pattern of deficits as that resulting from hippocampal damage.

The role of the hippocampus and surrounding cortices in the consolidation and retrieval of episodic memories is currently under debate (Jarrard, 2001; Murray and Bussey, 2001; Nadel and Moscovitch, 2001; Squire et al., 2001; Sutherland et al., 2001). Although a full review is beyond the scope of this report, clearly, additional study with careful attention to lesion methods, experimental design, and task selection is needed to clarify the role of the medial temporal lobe in remote memory. Regarding contextual representations, the available evidence suggests that the hippocampus has a time-limited involvement in associations of fear to context. Our studies show that the contribution of the PER and POR cortices to memory for context differs from that of the hippocampus, with the PER and POR having a longer-lasting role in processing contextual information. Although speculative, it could be that the hippocampus is involved in consolidating the representation for context into association cortex (PER and POR). Association cortex would then take over until the contextual representation is distributed across the relevant unimodal neocortical regions. It should be noted, however, that this interpretation may only apply to contextual representations. PER damage causes retrograde gradients in some memory tasks (Wiig et al., 1996) (but see Mumby and Glenn, 2000; Glenn et al., 2003). Likewise, damage to the hippocampus does not cause temporally graded retrograde amnesia in all tasks (Sutherland et al., 2001; Jonasson et al., 2004) (but see Winocur et al., 2001). Despite those caveats, the present findings indicate that the cortical regions surrounding the hippocampus and the hippocampus itself can be functionally dissociated and that consolidation theory does not apply equally to all medial temporal lobe structures.

\section{References}

Aggleton JP, Brown MW (1999) Episodic memory, amnesia, and the hippocampal-anterior thalamic axis. Behav Brain Sci 22:425-489.

Anagnostaras SG, Maren S, Fanselow MS (1999) Temporally graded retrograde amnesia of contextual fear after hippocampal damage in rats: within-subjects examination. J Neurosci 19:1106-1114.

Anagnostaras SG, Maren S, Fanselow MS (2001) Hippocampus and contextual fear conditioning: recent controversies and advances. Hippocampus 11:8-17.

Bucci DJ, Burwell RD (2004) Deficits in attentional orienting following damage to the perirhinal or postrhinal cortices. Behav Neurosci 118:1117-1122.

Bucci DJ, Phillips RG, Burwell RD (2000) Contributions of postrhinal and perirhinal cortex to contextual information processing. Behav Neurosci 114:882-894.

Bucci DJ, Saddoris MP, Burwell RD (2002) Contextual fear discrimination is impaired by damage to postrhinal or perirhinal cortex. Behav Neurosci 116:479-488.

Burwell RD, Amaral DG (1998a) Perirhinal and postrhinal cortices of the rat: interconnectivity and connections with the entorhinal cortex. J Comp Neurol 391:293-321.

Burwell RD, Amaral DG (1998b) Cortical afferents of the perirhinal, postrhinal, and entorhinal cortices. J Comp Neurol 398:179-205.

Burwell RD, Saddoris MP, Bucci DJ, Wiig KA (2004) Corticohippocampal contributions to spatial and contextual learning. J Neurosci 24:3826-3836.

Corodimas KP, LeDoux JE (1995) Disruptive effects of posttraining perirhi- 
nal cortex lesions on conditioned fear: contributions of contextual cues. Behav Neurosci 109:613-619.

Eichenbaum H (1997) Memory: old questions, new perspective. Curr Biol 7:R53-R55.

Eichenbaum H, Otto T, Cohen NJ (1994) Two functional components of the hippocampal memory system. Behav Brain Sci 17:449-518.

Fanselow MS (1980) Conditional and unconditional components of postshock freezing. Pavlov J Biol Sci 15:177-182.

Glenn MJ, Nesbitt C, Mumby DG (2003) Perirhinal cortex lesions produce variable patterns of retrograde amnesia in rats. Behav Brain Res 141:183-193.

Herzog C, Otto T (1997) Odor-guided fear conditioning in rats: 2. Lesions of the anterior perirhinal cortex disrupt fear conditioned to the explicit conditioned stimulus but not to the training context. J Neurosci 111:1265-1272.

Holland PC, Bouton ME (1999) Hippocampus and context in classical conditioning. Curr Opin Neurobiol 9:195-202.

Jarrard LE (2001) Retrograde amnesia and consolidation: anatomical and lesion considerations. Hippocampus 11:43-49.

Jonasson Z, Ballantyne JK, Baxter MG (2004) Preserved anterograde and retrograde memory of rapidly acquired olfactory discriminations after neurotoxic hippocampal lesions. Hippocampus 14:28-39.

Kim JJ, Fanselow MS (1992) Modality-specific retrograde amnesia of fear. Science 256:675-677.

Kosel KC, Van Hoesen GW, Rosene D (1983) A direct projection from the perirhinal cortex (area 35) to the subiculum in the rat. Brain Res 269:347-351.

Lindquist DH, Jarrard LE, Brown TH (2004) Perirhinal cortex supports delay fear conditioning to rat ultrasonic social signals. J Neurosci 24:3610-3617.

Maguire EA, Mummery CJ, Buchel C (2000) Patterns of hippocampalcortical interaction dissociate temporal lobe memory subsystems. Hippocampus 10:475-482.

Maren S, Aharonov G, Fanselow MS (1997) Neurotoxic lesions of the dorsal hippocampus and Pavlovian fear conditioning in rats. Behav Brain Res $88: 261-274$

Maren S, Anagnostaras SG, Fanselow MS (1998) The startled seahorse: is the hippocampus necessary for contextual fear conditioning? Trends Cogn Sci 2:39-42.

McClelland JL, McNaughten BL, O’Reilly RC (1995) Why are there complementary learning systems in the hippocampus and neocortex: insights from successes and failures of connectionist models of learning and memory. Psychol Rev 102:419-457.

Mumby DG, Glenn MJ (2000) Anterograde and retrograde memory for object discriminations and places in rats with perirhinal cortex lesions. Behav Brain Res 114:119-134.
Murray EA, Bussey TJ (2001) Consolidation and the medial temporal lobe revisited: methodological considerations. Hippocampus 11:1-7.

Naber PA, Caballero-Bleda M, Jorritsma-Byham B, Witter MP (1997) Parallel input to the hippocampal memory system through peri- and postrhinal cortices. NeuroReport 8:2617-2621.

Nadel L, Bohbot V (2001) Consolidation of memory. Hippocampus 11:56-60.

Nadel L, Moscovitch M (2001) The hippocampal complex and long-term memory revisited. Trends Cogn Sci 5:228-230.

Nadel L, Willner J (1980) Context and conditioning: a place for space. Physiol Psychol 8:218-228.

Paxinos G, Watson C (1998) The rat brain in stereotaxic coordinates, Ed 4. San Diego: Academic.

Rosen JB, Hitchcock JM, Miserendino MJD, Falls WA, Campeau S, Davis M (1992) Lesions of the perirhinal cortex but not of the frontal, medial prefrontal, visual, or insular cortex block fear-potentiated startle using a visual conditioned stimulus. J Neurosci 12:4624-4633.

Sacchetti B, Lorenzini CA, Baldi E, Tassoni G, Bucherelli C (1999) Auditory thalamus, dorsal hippocampus, basolateral amygdala, and perirhinal cortex role in the consolidation of conditioned freezing to context and to acoustic conditioned stimulus in the rat. J Neurosci 19:9570-9578.

Sacchetti B, Baldi E, Lorenzini CA, Bucherelli C (2002) Differential contribution of some cortical sites to the formation of memory traces supporting fear conditioning. Exp Brain Res 146:223-232.

Schulz B, Fendt M, Richardson R, Schnitzler HU (2004) Temporary inactivation of the perirhinal cortex by muscimol injections block acquisition and expression of fear-potentiated startle. Eur J Neurosci 19:713-720.

Shi CJ, Cassell MD (1997) Cortical, thalamic, and amygdaloid projections of rat temporal cortex. J Comp Neurol 382:153-175.

Squire LR, Zola-Morgan S (1991) The medial temporal lobe memory system. Science 253:1380-1386.

Squire LR, Clark RE, Knowlton BJ (2001) Retrograde amnesia. Hippocampus 11:50-55.

Sutherland RJ, Weisend MP, Mumby D, Astur RS, Hanlon FM, Koerner A, Thomas MJ, Wu Y, Moses SN, Cole C, Hamilton DA, Hoesing JM (2001) Retrograde amnesia after hippocampal damage: recent vs. remote memories in two tasks. Hippocampus 11:27-42.

Wiig KA, Cooper LN, Bear MF (1996) Temporally graded retrograde amnesia following separate and combined lesions of the perirhinal cortex and fornix in the rat. Learn Mem 3:313-325.

Winocur G, Rawlins JNP, Gray JA (1987) The hippocampus and conditioning to contextual cues. Behav Neurosci 101:617-625.

Winocur G, McDonald RM, Moscovitch M (2001) Anterograde and retrograde amnesia in rats with large hippocampal lesions. Hippocampus 11: $18-26$. 